\title{
Paper acceptance time in respiratory research: room for improvement?
}

\author{
To the Editor:
}

We recently submitted a paper to a top five respiratory journal in terms of average citations per article. The paper was swiftly sent for peer review. A month later, the review was in, and the paper was now "awaiting associate editor decision". After another 6 weeks, and at the time of writing this letter, the paper is still with the handling editor. Like us, many authors have probably at this stage speculated when it would be reasonable to contact the editorial office and enquire about the status.

A recent study carried out for Nature showed that the median time between submission and acceptance of a paper for 4375 different journals was approximately 100 days $[1,2]$. The study was based on an analysis of all papers in PubMed for which these data were listed from 1965 to 2015 and included more than 6 million observations of acceptance and publication time. Somewhat surprisingly, the acceptance time has remained fairly constant for more than 30 years and may in fact underestimate the actual time, as not all journals choose to deposit these data. Moreover, some journals use the re-submission date rather than the date for initial submission as the "start date", which may also bias the results.

Based on this large dataset at hand, available on GitHub [2], it is possible to analyse acceptance time for most respiratory journals. If the analysis is restricted to those respiratory journals with more than three citations per paper over 2 years, of which there are 22 journals [3], 18820 papers are found with reported acceptance times. All of these papers were published from 2000 to 2015 (both years included). Only 15 of the 22 journals have deposited acceptance data on PubMed (figure 1). The mean \pm SD and median (interquartile range) for acceptance time is $120 \pm 82$ and 105 (69-153) days, respectively. The average acceptance time for respiratory journals is consequently a little higher than the PubMed average. Interestingly, 2209 (12\%) of all respiratory papers had an acceptance time/delay of more than 200 days. Although there was a statistically significant difference between journals $(\mathrm{p}<0.001)$, all of them had a median acceptance time below 135 days. A total of 191 (1\%) of all papers had an acceptance time below 5 days, which may represent wrong time stamping in PubMed, or may comprise other types of papers, such as invited articles and editorials, that are not subject to peer review. A very quick acceptance time may also represent a revised manuscript being resubmitted, rather than a new submission, as journals may "reset the clock" in some cases.

Acceptance time was also assessed as a function of calendar year in a linear regression model [4]. Acceptance time increased by $0.31 \%$ per year $(p=0.07)$ which essentially implies that acceptance time has remained fairly constant for the past 15 years. Mean \pm SD and median (interquartile range) publication time, i.e. time from acceptance to online publication, is $44 \pm 44$ and 34 (16-56), respectively. In contrast to acceptance time, publication time has decreased by $8.2 \%$ per year $(\mathrm{p}<0.001)$ over the same period.

A limitation to these analyses is that only submission and acceptance dates are deposited on PubMed. A better quality indicator would be the decision time, i.e. time from first submission to a final decision. Even though most journals will have data on decision time, only the authors would know the complete submission history of a specific paper. Thus, we believe that more data is still needed to inform the debate on the peer review system, and journals could consider making more data on the publication process available.

A few attempts have been made to accelerate dissemination of research in science. Some authors have used platforms such as bioR $\chi$ iv operated by Cold Spring Harbor Laboratory, Cold Spring Harbor, NY, USA (biorxiv. org) to make their unpublished and un-refereed "preprints" immediately available to the scientific community in real time. This may be done prior to publication in a journal. Researchers may also display data, and their metadata, using open access data repositories. Such a repository is a virtual archive to collect, disseminate and

@ERSpublications

This letter analyses paper acceptance time in respiratory journals and discusses novel ways to disseminate research http://ow.ly/b9uX306esnA

Cite this article as: Ronit A, Vestbo J. Paper acceptance time in respiratory research: room for improvement? Eur Respir J 2017; 49: 1601951 [https://doi.org/10.1183/13993003.01951-2016]. 


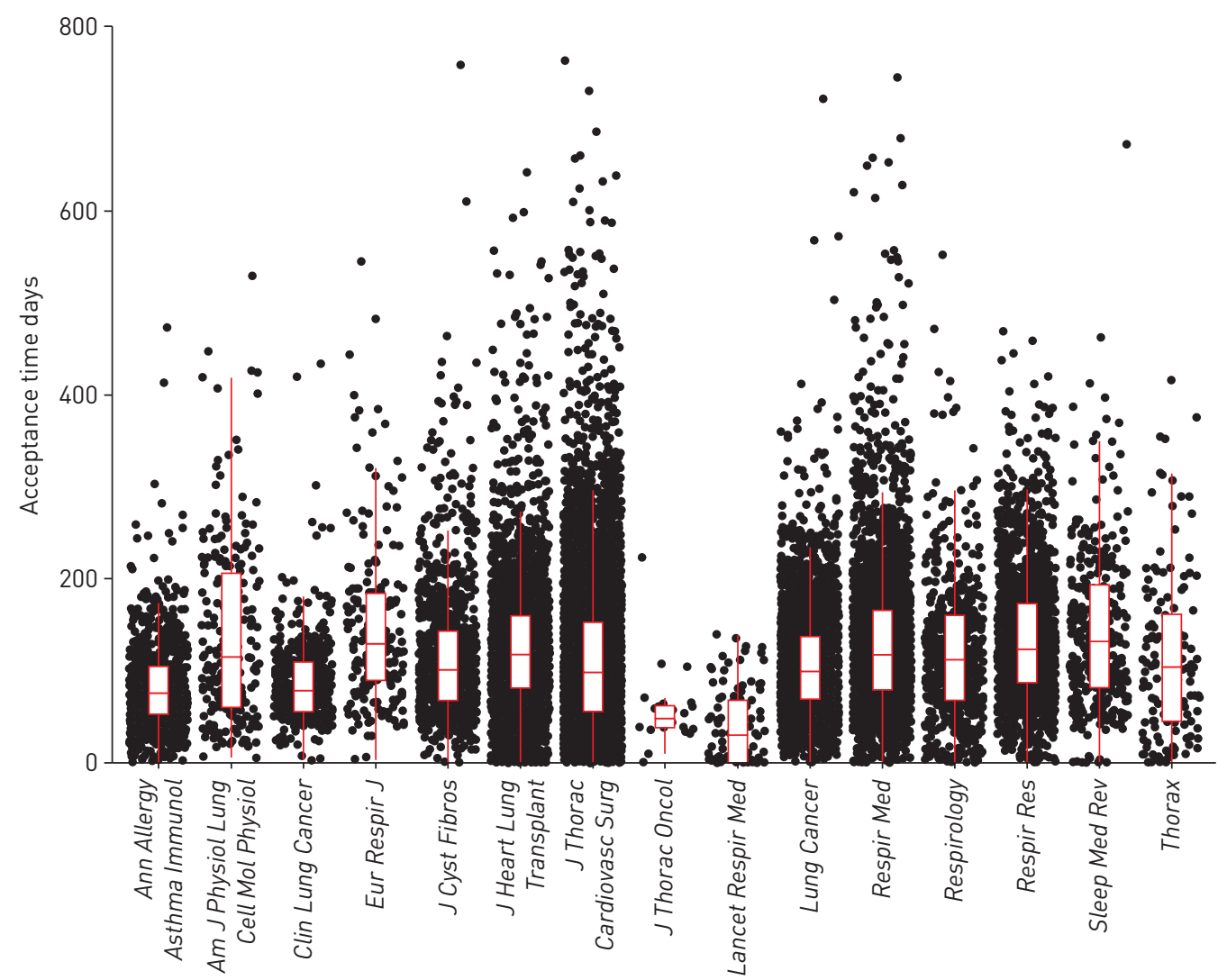

FIGURE 1 Acceptance time for respiratory journals. Acceptance time was calculated as time from submission to acceptance for 18805 papers from 2000 to 2015 (both years included). 15 data points were $>800$ days and omitted from graph. Ends of whiskers represent upper/lower quartile \pm 1.5 times the interquartile distance. Data from Am J Respir Crit Care Med, Am J Respir Cell Mol Biol, CHEST, Curr Opin Pulm Med, Eur Respir Rev, Int $J$ COPD and J Breath Res were not available. Anachronistic data and acceptance time of zero days were omitted from analysis. As data was heteroscedastic, a rank-based one-way ANOVA (i.e. Kruskal-Wallis test) was used to determine if acceptance time was statistically different across journals $(p<0.001)$. Log-transformed acceptance time was also modelled as a function of calendar year in a linear regression model (see text for results and note that log scale informs on relative changes (multiplicative)).

preserve data [5]. Importantly, a deposited dataset may also be assigned a digital object identifier (DOI) [6]. Some of these data repositories also work with a few journals to integrate data submission with article submission. Examples include DRYAD (datadryad.org), Figshare (figshare.com) and Zenodo (zenodo.org).

We are convinced that the editors and staff at all respiratory journals work very hard to lower acceptance/ rejection time. Importantly, some respiratory journals have also made a special effort to reduce decision time for the peer review system, including novel strategies to attract reviewers [7]. From the above analyses it is also evident that publication time has been lowered. We also believe that much delay is caused by researchers shopping around for journals with as high impact factor as possible for their manuscript. In fact journals with the highest and lowest impact factors may be associated with the longest acceptance times [8].

The peer review system has been in existence since The Philosophical Transactions of the Royal Society was first published more than 300 years ago [9]. It still serves to validate and uphold the overall quality of research [7, 10]. The Publishing Consortium surveyed the attitudes and perception of 3400 academics in relation to peer review [11]. In this report, $64 \%$ of academics declared themselves satisfied with the current system (a mere $12 \%$ were dissatisfied). However, peer review is also perceived to be slow by some ( $38 \%$ were dissatisfied with review times) and reviewers reported work overload [11]. With the increasing number of studies - and thus manuscripts - we believe it could be time to consider if the current system serves us well. It does not necessarily have to be either for or against the archetypical "old and slow peer review" versus the "sloppy and un-refereed" preprints/repositories. The two approaches discussed here may in fact be complementary, and if used together, they may be a tool to accelerate the dissemination of high quality respiratory research. While others consider this, we will patiently be waiting for the editor's decision, and try not to bother the editorial office with our impatience, as this may slow them down. 
Andreas Ronit ${ }^{1}$ and Jørgen Vestbo ${ }^{2}$

${ }^{1}$ Viro-immunology Research Unit, Dept of Infectious Diseases 8632, University Hospital Copenhagen, Copenhagen, Denmark. ${ }^{2}$ Division of Infection, Immunity and Respiratory Medicine, University of Manchester, Manchester, UK.

Correspondence: Andreas Ronit, Dept of Infectious Diseases 8632, University Hospital Copenhagen, Blegdamsvej 9, DK-2100 Copenhagen E, Denmark. E-mail: andreas.ronit@regionh.dk

Received: Sept 232016 | Accepted after revision: Oct 242016

Conflict of interest: Disclosures can be found alongside this article at erj.ersjournals.com

Acknowledgements: We would like to acknowledge D.S. Himmelstein (bioinformationist; University of Pennsylvania, Philadelphia, PA, USA) for allowing us to analyse data.

\section{References}

Powell K. Does it take too long to publish research? Nature 2016; 530: 148-151.

2 Himmelsein DS, Powell K. Analysis for "The History of Publishing Delays" blog post v1.0. https://zenodo.org/ record/45516/export/hx\#.WCyJTbKLSUn Date last updated: February 3, 2016. Date last accessed: September 5, 2016.

3 SCImago Journal and Country Rank. Pulmonary and Respiratory Medicine. www.scimagojr.com/journalrank.php? category $=2740$ Date last updated: September 5, 2016. Date last accessed: September 5, 2016.

4 R Core Team. R: A Language and Environment for Statistical Computing. Vienna, R Foundation for Statistical Computing, 2015.

5 OpenAIRE. FAQ/General Info. www.openaire.eu/support/faq. Date last updated: September 5, 2016. Date last accessed: September 5, 2016.

6 GitHub. Making Your Code Citable. https://guides.github.com/activities/citable-code/ Date last updated: May 15, 2014. Date last accessed: September 5, 2016.

$7 \quad$ Migliori GB, Soriane JB, Brusasco V, et al. ERJ peer reviewers: does this pillar of the Journal's quality need help? Eur Respir J 2011; 38: 251-252.

8 Quantixed. Some Things Last a Long Time. https:/quantixed.wordpress.com/2014/04/15/some-things-last-a-longtime/ Date last updated: April 15, 2014. Date last accessed: September 5, 2016.

9 The Royal Society Publishing. Philosophical Transactions: the World's First Science Journal. http://rstl. royalsocietypublishing.org/ Date last accessed: September 5, 2016.

10 Dinh-Xuan AT, Brusasco V. Refining the changes. Or, how to modify the shape, not the spirit. Eur Respir J 2009; 34: 1 .

11 Ware M, Monkman M. Peer review in schorlarly journals: Perspective of the scholarly community- an international study. London, Publishing Research Consortium, 2008; pp. 1-73. 